\author{
R.M. Peleshchak, O.V. Kuzyk, O.O. Dan'kiv
}

\title{
Formation of Nanoclusters on the Adsorbed Surface under the Action of Comprehensive Pressure and Electric Field
}

\author{
Ivan Franko Drohobych State Pedagogical University, Drohobych, 82100,Ukraine, e-mail: rpeleshchak@ukr.net
}

In the paper, the influence of the electric field and the comprehensive pressure on the conditions of formation and the period of the surface superlattice of adatoms in semiconductors is investigated. It is established that in GaAs semiconductor, an increase in the comprehensive pressure and the electric field strength, depending on the direction, leads to an increase or decrease of the critical temperature (the critical concentration of adatoms), at which the formation of self-organized nanostructure is possible. It is shown that in strongly alloyed n-GaAs semiconductor, the increase of the electric field strength leads to a monotonous change (decrease or increase depending on the direction of the electric field) of the period of self-organized surface nanostructures of adatoms. The period of nanometer structure of the adatoms depending on the value of comprehensive pressure, temperature, average concentration of the adatoms and conduction electrons is defined. It is established that the increase in pressure leads to expansion of temperature intervals within which nanometer structures of the adatoms are formed, and the decrease of their period.

Keywords: nucleation, comprehensive pressure, electric field, adatom, surface superlattice, deformation.

Article acted received 03.07.2019; accepted for publication 15.09.2019.

\section{Introduction}

Laser-induced periodic surface nanostructures can be generated on practically any material (metals, semiconductors, dielectrics) at irradiation with linearly polarized radiation and are formed in a wide range of intervals of impulses, ranging from continuous wave radiation to several femtoseconds [1-4]. In the experimental work [5] it was shown that their formation is caused by the effect of the long-range action of a laser pulse and is explained by the influence of the pressure gradient of the surface acoustic wave. The information on nucleation of periodic nanostructures of adsorbed atoms (adatoms) and implanted impurities is important for optimization of the technological process and predictable control of the physical parameters of semiconductor structures with nanoclusters.

In [4], the theory of spontaneous nucleation of the surface nanometer lattice which is due to instability in the system of adatoms interacting with self-consisted surface acoustic wave (SAW) was developed. Within this theory, the conditions of formation of nanoclusters on the surface of solids and the periods of nanometer lattice as a function of concentration of adatoms and temperature are defined.

In the experimental works $[6,7]$ the influence of the external electric field on the formation of self-organized nanostructures was investigated. In particular, in [7] it is shown that the external electric field increases the density and changes the size of the $\mathrm{CuO}$ quantum wires.

The elastic fields created by defects are the determining factor in the formation of the surface superlattice of adatoms. In works [8-12] it is shown that external hydrostatic pressure, ultrasonic wave and doping with isovalent impurities should contribute to improving the conditions for the formation of surface nanostructures.

In heteroepitaxial systems with a slight inconsistency in the lattice parameters of contacting materials, the growth regime of nanoclusters is determined only by the ratio of the energies of the two surfaces and the energy of the partition boundary. If the sum of the surface energy of the expanding layer and the energy of the partition boundary is less than the energy of the substrate surface, that is, if the expanding material moistens the substrate, then the Franco-van der Merwe growth regime arises [13]. The formation of strained QDs in a strained nanoheterosystem by the Stranski-Krastanov mechanism [14] occurs in two stages. In the first stage, a strained pseudomorphous InAs layer grows. As the layer reaches a critical thickness of 1.5-1.7 monolayers (MLs), the second stage begins: the pseudomorphous InAs layer is spontaneously decomposed into a system of crystalline 


$$
N_{0}<N_{c}
$$

laser irradiation
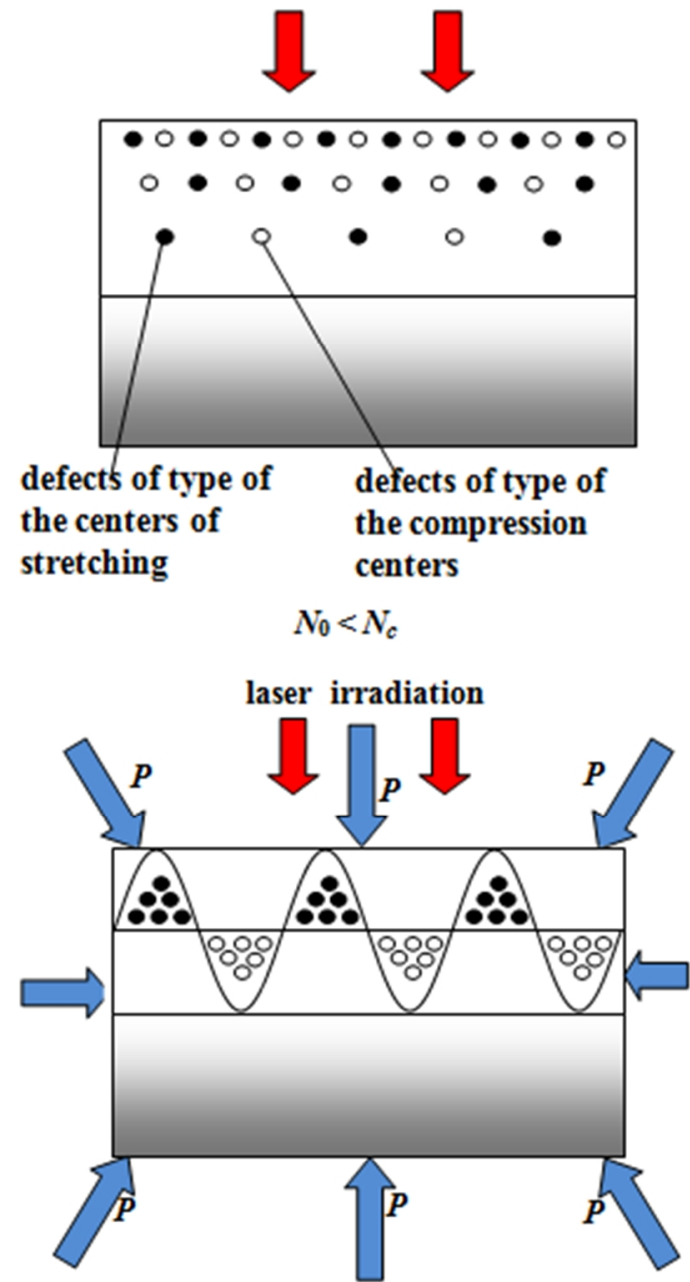

a)

$N_{0}>N_{c}$

laser irradiation
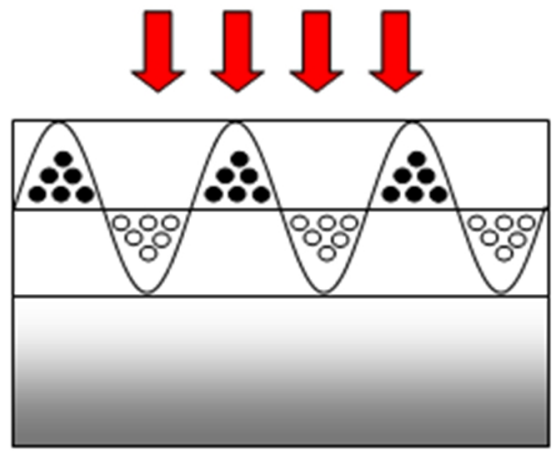

b)

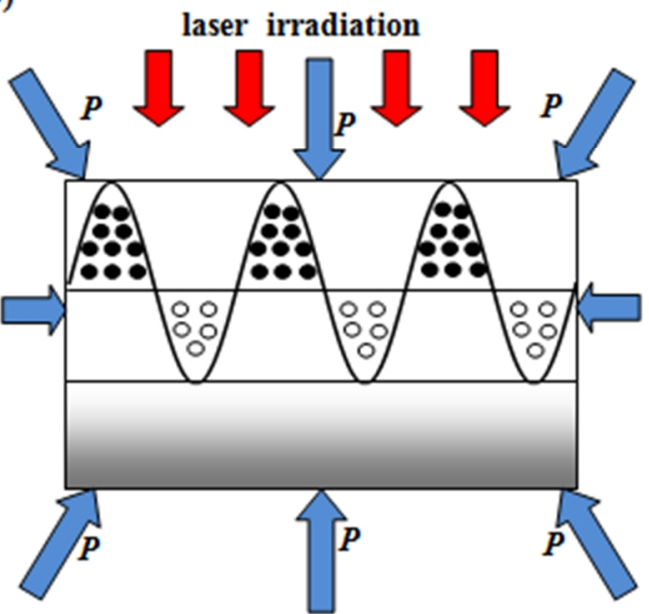

Fig. 1. The geometrical model of the near-surface layer of semiconductor, exposed to laser irradiation a) without the influence of external pressure; b) under the action of comprehensive pressure.
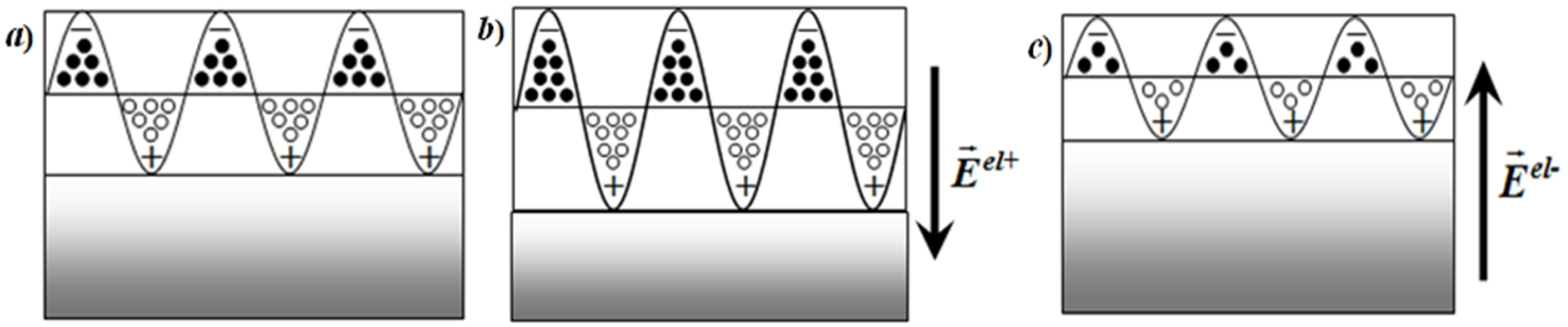

Fig. 2. The geometric model of formation of the surface superlattice of adatoms under the influence of the electric field.

QD islands and an InAs wetting layer with a thickness of about 1 ML. Such decomposition is induced by the relaxation of elastic strains produced in the heteroepitaxial system with a lattice mismatch and with different coefficients of thermal expansion for the GaAs substrate and the InAs epitaxial layer.
The deformation arising on the surface of semiconductor leads to the modulation of the bottom of the conduction band and, respectively, to the modulation of electronic density. The arising nonuniform electric field leads to the nonuniform displacement of the nodes of the crystal lattice and, respectively, to the change in 
the amplitude of the SAW $[15,16]$. Therefore, it can be expected that when placing a semiconductor in the external electric field, it is possible to change the conditions of the formation of laser-induced periodic surface nanostructures and predictably control their parameters due to the interaction of the electric field with nonuniform distributed on the surface of free current carriers. It is also clear that the action of external elastic fields will affect the conditions for the formation of nanoclusters [17-19]. In particular, in [9] the results of experimental studies of the external influence of mechanical deformation on the conditions for the formation of quantum dots are presented. It was shown in works [20-22] that an ultrasonic wave can be used to control the transport properties of semiconductors and change their defect structure. This possibility is associated with the diffusion processes of impurity atoms, the formation and decay of complexes, and the clustering of impurity atoms and intrinsic defects in periodic deformation fields.

In this paper the influence of the external electric field perpendicular to the SAW and the influence of comprehensive pressure on the nucleation of the nanometer periodic structure of adatoms in GaAs semiconductor is investigated.

\section{The model}

Let's consider a semiconductor that is exposed to laser irradiation, an action of a comprehensive pressure and / or an electric field (figure. 1,2). The equation for the displacement vector $\dot{u}$ of an elastic medium has the form [23]:

$$
\frac{\partial^{2} \stackrel{r}{u}}{\partial t^{2}}=c_{t}^{2} \Delta \stackrel{r}{u}+\left(c_{l}^{2}-c_{t}^{2}\right) \operatorname{grad}(\operatorname{div} \stackrel{r}{u})
$$

where $c_{l}$ and $c_{t}$ are longitudinal and transverse sound velocities, respectively.

Let the surface of semiconductor coincide with the plane $z=0$ (the $z$-axis is directed into the crystal depth), and assume that along the $x$-axis there is the surface perturbation of the elastic medium, which is given in the form of a static SAW which quickly fades into the depth of semiconductor and has an amplitude rowing with time [4]:

$$
\begin{gathered}
u_{x}=-i q R e^{i q x+\lambda t-k_{l} z}-i k_{t} Q e^{i q x+\lambda t-k_{t} z}, \\
u_{z}=k_{l} R e^{i q x+\lambda t-k_{l} z}+q Q e^{i q x+\lambda t-k_{t} z},
\end{gathered}
$$

where $k_{l, t}^{2}=q^{2}+\frac{\lambda^{2}}{c_{l, t}^{2}}, \lambda$ is the increment of defectdeformation instability; $R$ and $Q$ are SAW amplitudes.

Then, deformation $\varepsilon$ on semiconductor surface $(z=0)$ is of the form

$$
\varepsilon=\frac{\partial u_{x}}{\partial x}+\frac{\partial u_{z}}{\partial z}=-\frac{\lambda^{2}}{c_{l}^{2}} R e^{i q x+\lambda t} .
$$

Consider the case where the semiconductor contains impurities - ionized donors, free electrons and neutral adatoms. The electroneutrality condition will look as follows:

$$
n_{0}=N_{d}^{+},
$$

where $N_{d}^{+}$and $n_{0}$ are the surface concentration of ionized donors and the spatially homogeneous values of the surface concentration of electrons, respectively.

Periodic surface deformation leads to spatial nonuniform redistribution of adatoms $N(x)$, the modulation of the bottom of the conduction band and, respectively, to redistribution of the concentration of conduction electrons $n(x)$ and the electrostatic potential $\varphi(x)$ :

$$
\begin{gathered}
N(x)=N_{0}+N_{1}(x)=N_{0}+N_{1}(q) e^{i q x+\lambda t}, \\
n(x)=n_{0}+n_{1}(x)=n_{0}+n_{1}(q) e^{i q x+\lambda t}, \\
\varphi(x)=\varphi(q) e^{i q x+\lambda t},
\end{gathered}
$$

where $N_{1}(q), n_{1}(q), \varphi(q)$ are the amplitudes of the corresponding periodic perturbations; $N_{0}$ is the spatially homogeneous values of the surface concentration of adatoms.

The Poisson equation, taking into account (5), (7) and (8), will take the form:

$$
-q^{2} \varphi(q)=\frac{e}{\varepsilon_{0} \tilde{\varepsilon} a} n_{1}(q),
$$

where $\varepsilon_{0}, \tilde{\varepsilon}$ are dielectric constant and dielectric permittivity of the medium, respectively.

The equations for concentration of adatoms can be presented as follows:

$$
\frac{\partial N}{\partial t}=D_{d} \frac{\partial^{2} N}{\partial x^{2}}-D_{d} \frac{\theta_{d}}{k_{B} T} \frac{\partial}{\partial x}\left(N \frac{\partial}{\partial x}\left(\varepsilon+l_{d}^{2} \frac{\partial^{2} \varepsilon}{\partial x^{2}}\right)\right),
$$

where $D_{d}$ is the surface diffusion coefficient; $k_{B}$ is Boltzmann constant; $T$ is temperature; $\theta_{d}$ is the deformation potential; $l_{d}$ is the characteristic length of interaction of adatoms with lattice atoms. The second term expresses the interaction of adatoms with the deformation field, taking into account the nonlocal interaction [4]. The defect which enters the surface of semiconductor leads to a change in its volume and energy, and the initial fluctuation of deformation under certain conditions causes the emergence of deformationinduced flows of adatoms. In nonuniform deformationconcentration field there are forces proportional to gradients of concentration and deformation.

Taking into account (4), (7), (8), and in the approximation of $N_{1} \ll<N_{0}$, the equation (10) is written:

$$
\lambda N_{1}(q)=-D_{d} q^{2} N_{1}(q)-\frac{D_{d} N_{0} \theta_{d}}{k_{B} T}\left(\frac{\lambda^{2}}{c_{l}^{2}} R q^{2}\left(1-q^{2} l_{d}^{2}\right)\right)
$$

The density of the electron current:

$$
j=-n \mu_{n} \frac{d \chi}{d x}
$$

where $j_{e l}$ is the flow of electrons; $\mu_{n}$ is the mobility of electrons; the electrochemical potential $\chi$ is defined by the relation

$$
\chi(x)=k_{B} T \ln \frac{n(x)}{N_{i}}-e \varphi(x)+a_{c} \varepsilon(x),
$$

where $N_{i}$ is the effective density of states; $N_{i}=2\left(2 \pi m k T / h^{2}\right)^{3 / 2} ; a_{c}$ is the constant of hydrostatic deformation potential of the conduction band. Then, taking into account (12), (13), the continuity equation can be written in the form: 

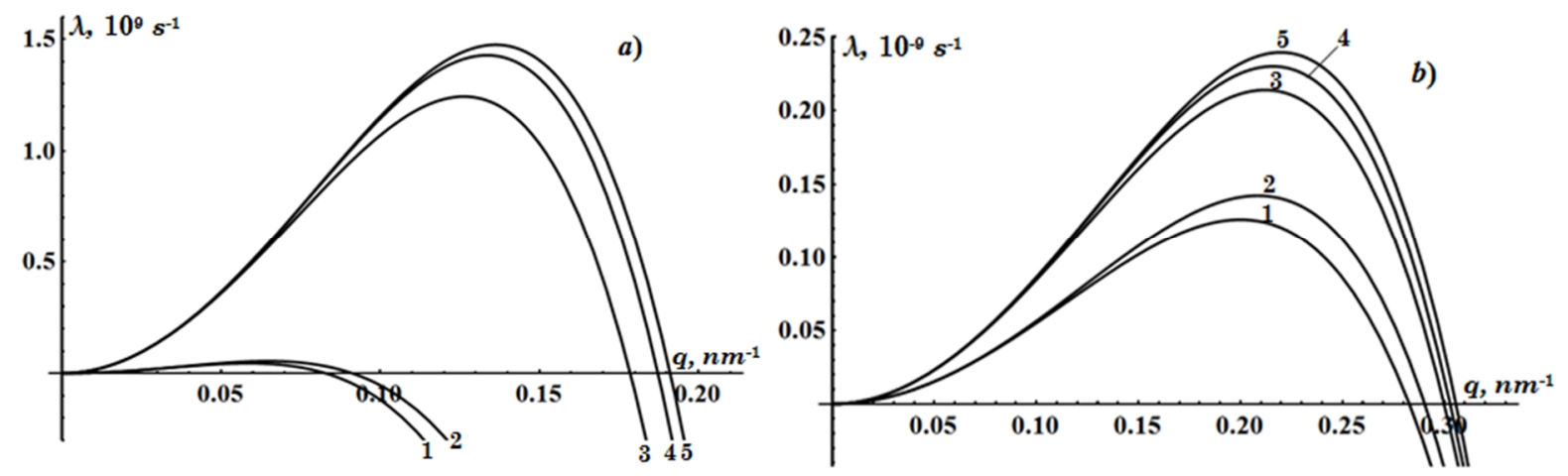

Fig. 3. Dependence of the increment of defect-deformation instability on the wave vector at the various values of the concentration of electrons and comprehensive pressure: $P_{0}=1.3 \operatorname{kbar}(1 ; 2) ; \quad P_{0}=2 \operatorname{kbar}(3 ; 4 ; 5) ; n_{0}=0(1 ; 3)$; $n_{0}=10^{11} \mathrm{~cm}^{-2}(4) ; \quad n_{0}=10^{13} \mathrm{~cm}^{-2}(2 ; 5) ; \quad$ a) $T=300 \mathrm{~K} ; \quad$ b) $T=100 \mathrm{~K}$
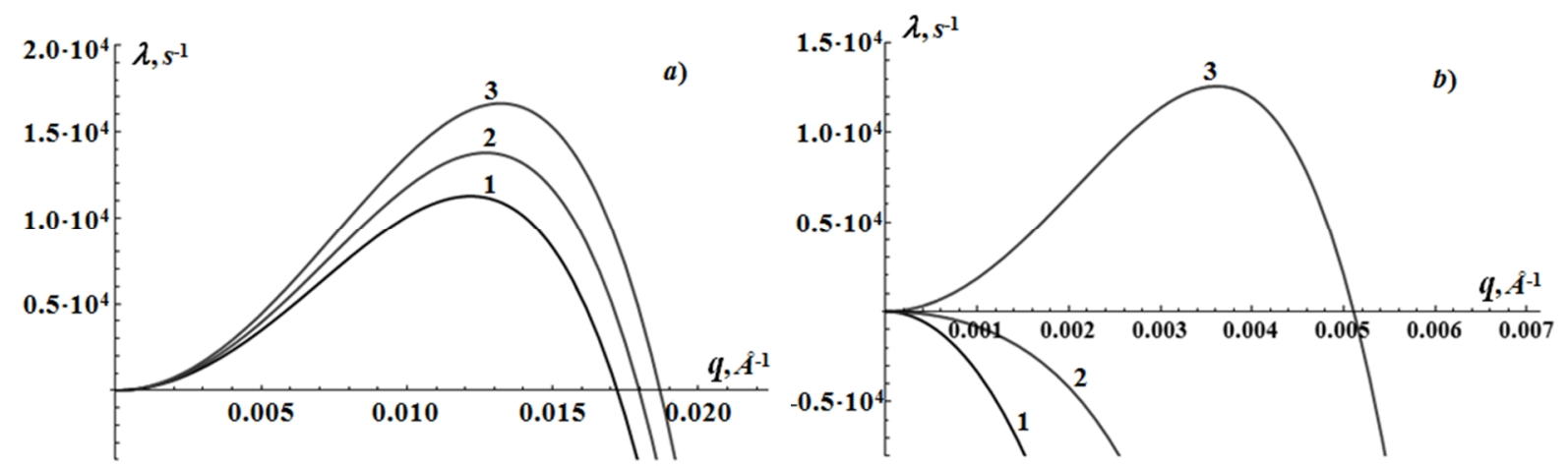

Fig. 4. Dependence of the increment of defect-deformation instability on the wave vector at the following values of parameters: $T=70 K(a) ; T=100 K(b) ; n_{0}=10^{12} \mathrm{~cm}^{-2}(a, b)$;

$\mathbf{1}-E^{e l}=0 ; \quad \mathbf{2}-E^{e l+}=30 \mathrm{kV} / \mathrm{cm} ; \quad \mathbf{3}-E^{e l+}=80 \mathrm{kV} / \mathrm{cm}$

$$
e \frac{\partial n}{\partial t}=k_{B} T \mu_{n} \frac{\partial}{\partial x}\left(n \frac{\partial}{\partial x} \ln \frac{n}{N_{i}}\right)-e \mu_{n} \frac{\partial}{\partial x}\left(n \frac{\partial \varphi}{\partial x}\right)+a_{c} \mu_{n} \frac{\partial}{\partial x}\left(n \frac{\partial \varepsilon}{\partial x}\right)
$$

Taking into account (4), (7) - (9), the equation (14) can be presented as follows:

$$
n_{1}(q)\left(e \lambda+k_{B} T \mu_{n} q^{2}+\frac{e^{2} n_{0} \mu_{n}}{\varepsilon_{0} \tilde{\varepsilon} a}\right)=a_{c} n_{0} \mu_{n} q^{2} \frac{\lambda^{2}}{c_{l}^{2}} R .
$$

Thus, on the surface of semiconductor, there is not only a periodic modulation of the surface relief with the accumulation of adatoms in the maxima or minima of deformation (depending on the sign of the deformation potential $\theta_{d}$ ), but also the surface modulation of the electronic density (figure 2, a). As can be seen from the formulas (4), (6), (7), the periodically distributed deformation $\varepsilon(x)$, the surface concentration of adatoms $N_{1}(x)$ and the concentration of electrons $n_{1}(x)$ are in the same phase at $\theta_{d}>0$ (the condition $q l_{d}<1$ is fulfilled) and $a_{c}<0$. For $\theta_{d}<0$ the distribution of the surface concentration of the adatoms $N_{1}(x)$ and the concentration of electrons $n_{1}(x)$ are in the opposite phase. Moreover, since for GaAs, the constant of the hydrostatic deformation potential of the conduction band $a_{c}<0$, an excess of electrons will be observed in those areas of the surface where accumulates adatoms, which are the centers of stretching, and conversely, in those areas of the surface where accumulates adatoms, which are the centers of compression, there will be a shortage of electrons in comparison with the mean value (figure 2, a). That is, the defects of the type of the centers of stretching and the electrons accumulate in deformation maxima. And, on the contrary, the defects of the type of the centers of compression accumulate in deformation minima and there is a decrease in the concentration of electrons in comparison with their spatially uniform value.

By placing the semiconductor in the electric field, perpendicular to the direction of propagation of the acoustic wave (figure 2, b, c), we create additional 
pressure on the surface of semiconductor, which, taking into account the hydrostatic pressure $P_{0}$, has the form:

$$
\sigma_{z z}=e n(x) E^{e l}-P_{0} \text {. }
$$

Moreover, in the case where the vector of the electric field strength $E^{e l}$ is directed into the crystal depth (figure 2, b), the surface area where the adatoms accumulate, which are the centers of stretching, and, respectively, electrons accumulate, is exposed to additional stretching. And the surface area in which the adatoms of type of the compression centers accumulate and there is a shortage of electrons, is exposed to additional compression. This, in turn, leads to the emergence of additional deformation-diffusion flow of adatoms (for defects of type of the centers of stretching in the direction of increasing deformation, for defects of type of the compression centers - in the opposite direction). At change of the direction of the electric field (figure 2, c), the additional pressure caused by the external electric field leads to the decrease in the deformation gradients on the surface of semiconductor and, accordingly, to the delocalization of adatoms.

The spatial nonuniform distribution of adatoms modulates the surface energy $F(x)$, which leads to the appearance of lateral mechanical tension $\sigma_{x z}=\partial F(N(x)) / \partial x$, which is compensated by shift tension in the medium [4]:

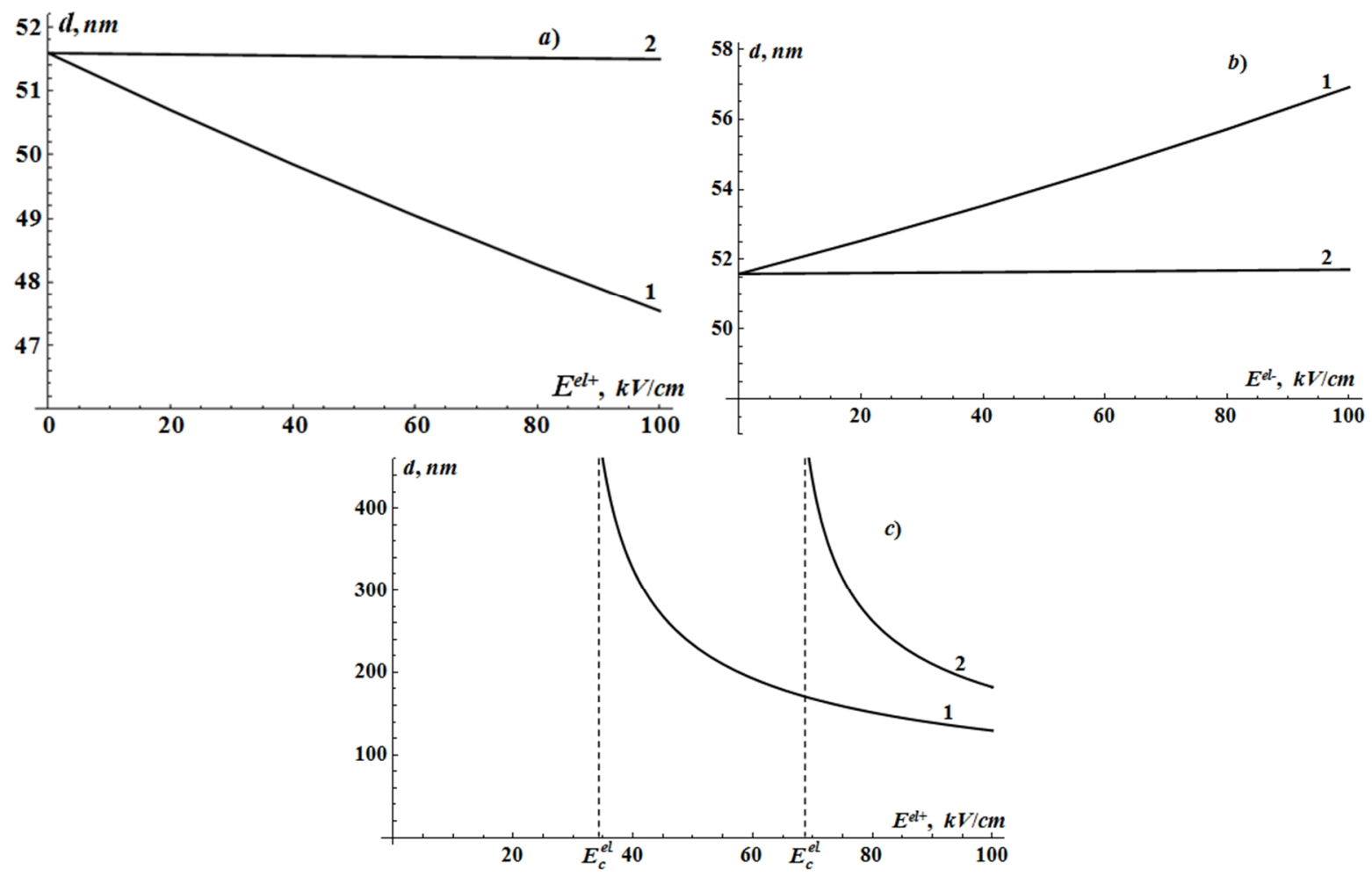

Fig. 5. Dependence of the period of the surface defect-deformation structure on the electric field strength at its various directions and at various values of the electron concentration: $T=70 K(a, b) ; \quad T=100 K(c) ; \quad 1-n_{0}=10^{12} \mathrm{~cm}^{-2} ; 2-n_{0}=10^{9} \mathrm{~cm}^{-2}$

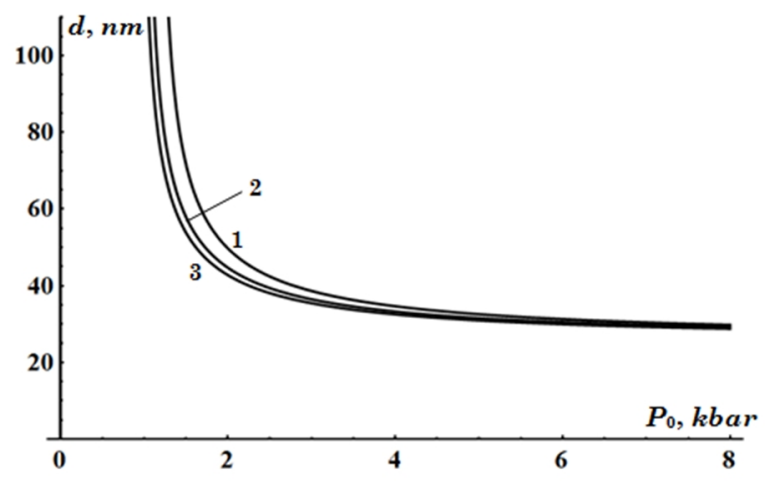

Fig. 6. Dependence of the period of the surface defect-deformation structure on the comprehensive pressure at various values of the electron concentration $(T=100 \mathrm{~K})$ :

$$
1-n_{0}=0 ; 2-n_{0}=10^{11} \mathrm{~cm}^{-2} ; 3-n_{0}=10^{13} \mathrm{~cm}^{-2}
$$




$$
F(N(x)) \approx F\left(N_{0}\right)+\left.\frac{\partial F}{\partial N}\right|_{N=N_{0}} N_{1}(x),\left.\frac{\partial F}{\partial N}\right|_{N=N_{0}} \approx \frac{\theta_{d}^{2} N_{0}}{a K}[24] .
$$

The boundary condition expressing the balance of lateral tensions is as follows:

$$
\frac{E}{2(1+v)}\left(\frac{\partial u_{x}}{\partial z}+\frac{\partial u_{z}}{\partial x}\right)_{z=0}=\frac{\partial F(N(x))}{\partial x}=\frac{\partial F}{\partial N} \frac{\partial N_{1}(x)}{\partial x},
$$

where $E$ and $v$ are Young's modulus and Poisson's ratio, respectively.

Besides, the interaction of adatoms with atoms of semiconductor results in the emergence of the normal mechanical tension on the surface, and the corresponding boundary condition is of the form:

$$
\frac{E(1-v)}{(1+v)(1-2 v)}\left(\frac{\partial u_{z}}{\partial z}+\frac{v}{1-v} \frac{\partial u_{x}}{\partial x}\right)_{z=0}=\frac{\theta_{d}}{a} N_{1}(x)+e n(x) E-P_{0}
$$

In order to get rid of the nonuniformity in the boundary condition (20), we will make a replacement:

$$
N_{1}(x, q)=\frac{a}{\theta_{d}}\left(P_{0}+P(q) e^{i q x+\lambda t}\right) .
$$

Thus, the system of homogeneous linear equations (17) and (18) for amplitudes $R$ and $Q$ is obtained and the dispersion dependencies $\lambda(q)$ can be obtained from the condition of non-triviality of solutions (from the condition of equality to zero of the determinant of this system).

\section{Calculation results and their discussion}

The calculations of $\lambda(q)$ were carried out for GaAs semiconductor at the following values of parameters: $N_{0}=2 \cdot 10^{12} \mathrm{~cm}^{-2} ; \quad l_{d}=2,9 \mathrm{~nm} ; \quad a=0,565 \mathrm{~nm} ;$ $c_{l}=4400 \mathrm{~m} / \mathrm{s} ; \quad c_{t}=2475 \mathrm{~m} / \mathrm{s} ; \quad \rho=5320 \mathrm{~kg} / \mathrm{m}^{3} ; \quad a_{c}=-$ $7,17 \mathrm{eV} \quad[25] ; \quad D_{d}=5 \cdot 10^{-9} \mathrm{~cm}^{2} / \mathrm{s} \quad[26] ; \quad \theta_{d}=10 \mathrm{eV}$; $\tilde{\varepsilon}=12 ; \quad \mu_{n}=8 \cdot 10^{3} \mathrm{~cm}^{2} / \mathrm{V} \cdot \mathrm{s}$. Mobility of electrons as function of temperature and concentration was determined by the technique given in work [27].

In figure 3 shows the results of calculation of the dependence of the increment of defect-deformation instability on the module of the wave vector at various values of the concentration of electrons, comprehensive pressure and $T$.

In figure 4 shows the results of calculation of the dependence of the increment of defect-deformation instability on the module of the wave vector at various values of the concentration of conduction electrons, temperature and electric field strength $\left(E^{e l+}\right)$ with the direction shown in figure $2, b$. Such dependence has a maximum, which is shifted towards great values of the module of the wave vector with the increase in the electric field strength. The formation of the surface superlattice of adatoms is possible only at positive values of the increment of defect-deformation instability $\lambda$. As seen from figure 4 , the formation of the superlattice is possible only at a temperature lower than a certain critical value $T_{c}$. The formation of the surface superlattice is defined by the ratio between the ordinary diffusion flow of adatoms (the first term of equation (10)) and the deformation flow (the second term of equation (10)). At high temperatures, the first term is decisive and over time the concentration of adatoms on the surface is levelled, and the formation of the surface superlattice is impossible $(\lambda<0)$. As the temperature decreases, the contribution of the deformation flow of adatoms increases, which becomes decisive at values of temperature less than the critical value of $T_{c}$. In this case, the defects accumulate in the maxima (minima) of deformation and the surface superlattice is formed. In particular, at a temperature of $100 \mathrm{~K}$ (figure $4, b$ ), in the absence of the electric field, the formation of the surface superlattice at a given intensity of laser irradiation is impossible. But the increase in the electric field strength leads to a change in the sign of the increment of defectdeformation instability $\lambda$ (figure $4, b$, curve 3 ), which makes it possible the formation of the superlattice of adatoms.

The value of $q_{\max }$, at which the increment of defectdeformation instability has maximum, determines the period of the dominant structure $d=2 \pi / q_{\max }$ (figure 5, $6)$. In figure 5,6 shows the dependence of the period of the surface superlattice of adatoms on the electric field strength (figure 5) and on the comprehensive pressure (figure 6) at various values of the electron concentration, temperature and various directions of the electric field. An increase in the electric field strength leads to a decrease (figure 5, $a, c$ ) and increase (figure 5,b) of the period of the surface superlattice of adatoms, depending on the direction of the electric field. The influence of the electric field on the period of the surface defectdeformation structure is manifested only in strongly alloyed semiconductors. In particular, in GaAs semiconductor with the surface electron concentration of $n_{0}=10^{12} \mathrm{~cm}^{-2}$, at a temperature of $70 \mathrm{~K}$, when the electric field strength increases to $60 \mathrm{kV} / \mathrm{cm}$, the period of the superlattice is changed by $2.5 \mathrm{~nm}$. And in semiconductor with the surface electron concentration of $n_{0}=10^{9} \mathrm{~cm}^{-2}$ the period of the superlattice practically does not change (figure 5, $a, b$ ).

At temperatures that are insignificantly higher than the critical temperature $T_{c}$ (figure $5, c$ ), there is a significant influence of the electric field, and the direction of the electric field $\left(E^{e l+}\right)$ corresponds to figure $2, b$. In this case, in the absence of the electric field, the defect-deformation structures do not arise at the given intensity of laser irradiation. However, there is some critical value of the electric field strength $E_{c}^{e l}$, at excess of which the formation of the surface periodic structure of adatoms is possible. Or it can be interpreted as expansion of temperature intervals under the action of the electric field, within which the formation of the surface superlattice is possible.

Changing the direction of the electric field (figure $2, c$ ) leads to deterioration of the conditions of the formation of self-organized nanostructures, irrespective of the sign of the deformation potential $\theta_{d}$, in particular to a decrease in the critical temperature and an increase in the period of the surface superlattice (figure 5, $b$ ). This is explained by the fact that in this case, the local deformation caused by the action of the electric field has 
the opposite character to the deformation created by adatoms (figure 2). The intensity of laser irradiation (the average concentration of adatoms) is another parameter that determines the conditions for the nucleation of the surface superlattice of adatoms. There is a critical value of the concentration of adatoms $N_{c}$. For concentrations less than $N_{c}$, the formation of the periodic surface structure is impossible. The comprehensive pressure and the electric field, the direction of which corresponds to figure $1, b$, allows to reduce the critical value of the concentration of adatoms (the intensity of laser irradiation) or increase the temperature below which the formation of the surface superlattices occurs [28]. The influence of the electric field is more significant for highly doped semiconductors.

In the framework of this model, the initial stage of the surface superlattice formation is considered. As a result of an increase in the amplitude of deformation, nonlinear effects become significant, which leads to its saturation (the amplitude no longer increases). In this case, for the calculation of the amplitude in the energy of elastic interaction, it is necessary to take into account the anharmonic terms $[24,29]$, or to consider the nonlinearity in the boundary conditions.

\section{Conclusions}

1. The theory of nucleation of the surface superlattice of adatoms in GaAs semiconductor under the influence of laser irradiation at the action of mechanical field (comprehensive pressure) and electric field, directed perpendicular to the direction of propagation of the SAW, is developed. The proposed theory takes into account the interaction of adatoms and conduction electrons with self-consistent SAW. The semiconductor can be located both in the external electric field and internal, created, for example, by a hetero-borders. The electric field, transverse to the direction of propagation of the SAW, creates an additional non-uniform mechanical tension. And depending on the direction of the electric field, it is possible to increase or decrease the deformation flows of adatoms.
2. The theory of nucleation of nanometer structure of the adatoms under the action of comprehensive pressure taking into account acousto-electronic interaction is developed. Within this theory the influence of comprehensive pressure and doping degree of semiconductor on the conditions of formation and the period of nanometer structure of the adatoms is investigated. It is established that the increase in pressure leads to expansion of temperature intervals within which nanometer structures of the adatoms are formed, and the decrease of their period.

3. The formation of the superlattice is possible if the average concentration of adatoms exceeds a certain critical value (or the temperature is less than a certain critical value). The concentration of adatoms is defined by the intensity of laser irradiation. It is established that in GaAs semiconductor, an increase in the electric field strength, depending on the direction, leads to an increase or decrease of the critical temperature (the critical concentration of adatoms), at which the formation of self-organized nanostructure is possible.

4. It is shown that the influence of the mechanical and the electric field is more significant in the highly doped semiconductors and also in semiconductors with a high value of the constant of hydrostatic deformation potential of the conduction band and electron mobility. $\mathrm{GaAs}$ an $\mathrm{Ge}$ is the most optimal for these parameters.

5. It is shown that in strongly alloyed n-GaAs semiconductor, the increase of the electric fields strength leads to a monotonous change (decrease or increase depending on the direction of the electric field) of the period of self-organized surface nanostructures of adatoms.

6. It is established that the influence of the electric field on the conditions of formation and the period of the surface superlattice does not depend on the sign of the deformation potential of adatoms.

Peleshchak R.M. - Professor, Doctor of Science (Physics), Head of the Department of Physics;

Kuzyk O.V. - PhD, Associate Professor, Associate Professor of the Department of Physics;

Dan'kiv O.M. - PhD, Associate Professor, Associate Professor of the Department of Physics.

[1] S. Höhm, M. Rohloff, A. Rosenfeld, J. Krüger, J. Bonse, Appl. Phys. A $\quad$ 110(3), 553 (2013) (doi: https://doi.org/10.1007/s00339-012-7184-z).

[2] J. Wu., Y. Yang, H. Gao, Y. Qi, AIP Advances 7 (3), 035218 (2017) (doi: https://doi.org/10.1063/1.4979507).

[3] J. Bonse, S. Höhm, S.V. Kirner, A. Rosenfeld, J. Krüger, IEEE Journal of selected topics in quantum electronics 23 (3), 9000615 (2017) (doi: https://doi.org/10.1109/JSTQE.2016.2614183).

[4] V.I. Emel'yanov, Laser Phys. 18(12), 1435 (2008) (doi: https://doi.org/10.1134/S1054660X08120104).

[5] A.I. Vlasenko, A. Baidullaeva, V.P. Veleschuk, P.E. Mozol, N.I. Boiko, O.S. Litvin, Semiconductors 49 (2), 229 (2015) (https://doi.org/10.1134/S1063782615020220).

[6] Y. Zeng, B. Tao, J. Phys. D: Appl. Phys. 49, 195308 (2016) (doi: doi.org/10.1088/0022-3727/49/19/195308).

[7] C. Tang, X. Liao, W. Zhong, H. Yu, Zh. Liu, RSC Advances 11, 6439 (2017) (doi: 10.1039/C6RA27426A).

[8] R.M. Peleshchak, O.V. Kuzyk, O.O. Dan'kiv, Journal of Nano- and Electronic Physics 10(1), 01014 (2018) (doi: https://doi.org/10.21272/jnep.10(1).01014).

[9] C. Taylor, E. Marega, E.A. Stach, G. Salamo, L. Hussey, M. Munoz, A. Malshe, Nanotechnology 19, 015301 (2008).(doi: https://doi.org/10.1088/0957-4484/19/01/015301). 
[10] R.M. Peleshchak, O.V. Kuzyk, O.O. Dan'kiv, $\quad$ Ukr. $\quad$ J. $\quad$ Phys. $\quad 61(8), \quad 741 \quad$ (2016) (doi: https://doi.org/10.15407/ujpe61.08.0747).

[11] R.M. Peleshchak, O.V. Kuzyk, O.O. Dan'kiv， J. Nano- Electron. Phys. 8(2), 02014 (2016) (doi: http://dx.doi.org/10.21272/jnep.8(2).02014).

[12] R.M. Peleshchak, S.K. Guba, O.V. Kuzyk, I.V. Kurilo, O.O. Dan'kiv, Semiconductors 47(3), 349 (2013) (doi: https://doi.org/10.1134/S1063782613030196).

[13] R. D. Vengrenovich, Yu. V. Gudyma, and S. V. Yarema, Semiconductors 35(12), 1378 (2001) (doi: https://doi.org/10.1134/1.1427975)).

[14] N.N. Ledentsov, V.M. Ustinov, V.A. Shchukin, P.S. Kop'ev, Zh.I. Alferov, D. Bimberg, Semiconnductors 32(4), 343 (1998) (doi: https://doi.org/10.1134/1.1187396).

[15] R.M. Peleshchak, I.I. Lazurchak, O.V. Kuzyk, O.O. Dan'kiv, G.G. Zegrya, Semiconductors 50(3), 314 (2016) doi: (https://doi.org/10.1134/S1063782616030180).

[16] R.M. Peleshchak, O.V. Kuzyk, O.O. Dan'kiv, $\quad$ Cond. $\quad$ Mat. $\quad$ Phys. $\quad 18(4), 43801 \quad$ (2015) (10.5488/CMP.18.43801).

[17] Ya. M. Olikh, M. D. Tymochko, O. Ya. Olikh, V. A. Shenderovsky, Journal of Electronic Materials 47, 4370 (2018) (https://www.springerprofessional.de/journal-of-electronic-materials-8-2018/15902282).

[18] C. Kristukat, A.R. Goci, Phys. stat. sol. (b) 244, 53 (2007) (doi: https://doi.org/10.1002/pssb.200672511).

[19] O.V. Balaban, I.I. Grygorchak, R.M. Peleshchak, O.V. Kuzyk, O.O. Dan'kiv, Progress in Natural Science: Mater. International 24(4), 397 (2014) (doi: https://doi.org/10.1016/j.pnsc.2014.07.003).

[20] S. Ostapenko, Appl. Phys. A 69(2), 225 (1999) (doi: 10.1007/s003390050994)..

[21] O.Ya. Olikh, K.V. Voytenko, R.M. Burbelo, Journal of Applied Physics 117(4), 044505 (2015) (doi: https://doi.org/10.1063/1.4906844).

[22] И. В. Островский, А. Б. Надточий, А. А. Подолян, Физ. техн. полупр. 36, 389 (2002).

[23] L.D. Landau, E.M. Lifshitz, Theory of Elasticity (Pergamon Press, London, 1970).

[24] R.M. Peleshchak, O.V. Kuzyk, O.O. Dan'kiv, Cond. Mat. Phys. 17(2), 23601 (2014) (doi: https://doi.org/10.5488/CMP.17.23601).

[25] C.G. van de Walle, Phys. Rev. B. 39(3), 1871 (1989) (doi: https://doi.org/10.1103/PhysRevB.39.1871).

[26] J.F. Wager, J. Appl. Phys. 69(5), 3022 (1991) ( doi: doi.org/10.1063/1.348589).

[27] T.T. Mnatsakanov, M.E. Levinshtein, Semiconductors, 38(1), 56 (2004) (doi: doi.org/10.1134/1.1641133).

[28] K.F. MacDonald, V.A. Fedotov, Applied Phys. Let. 80, 1643 (2002) (doi: https://doi.org/10.1063/1.1456260).

[29] R.M. Peleshchak, O.V. Kuzyk, O.O. Dan'kiv, Ukr. J. Phys. 55(4), 434 (2010).

Р.М. Пелещак, О.В. Кузик, О.О. Даньків

\title{
Формування нанокластерів на адсорбованій поверхні під впливом всебічного тиску та електричного поля
}

\author{
Дрогобицький державний педагогічний університет імені Івана Франка, м. Дрогобич 82100, Украӥна, \\ e-mail: rpeleshchak@ukr.net
}

\begin{abstract}
У роботі досліджено вплив електричного поля та всебічного тиску на умови формування та період поверхневої надгратки адсорбованих атомів у напівпровідниках. Встановлено, що у напівпровіднику GaAs збільшення всебічного тиску та напруженості електричного поля залежно від напрямку призводить до збільшення або зменшення критичної температури (критичної концентрації адатомів), при якій можливе формування самоорганізованої наноструктури. Показано, що у сильнолегованому напівпровіднику n-GaAs збільшення напруженості електричного поля призводить до монотонної зміни (зменшення чи збільшення залежно від напрямку електричного поля) періоду самоорганізованих поверхневих наноструктур адатомів. Визначено період нанометрової структури адатомів залежно від величини всебічного тиску, температури, середньої концентрації адатомів та електронів провідності. Встановлено, що збільшення тиску призводить до розширення температурних інтервалів, у межах яких формуються нанометрові структури адатомів, та зменшення їх періоду.

Ключові слова: нуклеація, всебічний тиск, електричне поле, адатом, поверхнева надгратка, деформація.
\end{abstract}

